\title{
Diagnostic Work Up in A Patient with A Progressive Cardio-/Encephalomyopathy, Peripheral Neuropathy and Respiratory Stridor
}

\author{
Manuela Zlamy*1, Sabine Scholl-Bürgi ${ }^{1}$, Benjamin Hetzer ${ }^{1}$, Herta Zellner ${ }^{1}$, Thomas Karall' ${ }^{1}$, Matthias \\ Baumannn ${ }^{1}$, Johannes Mayr ${ }^{3}$, Reka Kovacs-Nagy ${ }^{2}$, Saskia Wortmann ${ }^{2,3}$ and Daniela Karall ${ }^{1}$ \\ ${ }^{1}$ Department of Pediatrics, Medical University of Innsbruck, Austria
}

${ }^{2}$ Institute of Human Genetics, Technical University Munich, Munich, Germany

${ }^{3}$ Department of Pediatrics, Salzburger Landeskliniken (SALK) and Paracelsus Medical University (PMU)

Received: May 30, 2018; Published: June 12, 2018

*Corresponding author: Manuela Zlamy, Clinic for Pediatrics I, Department of Pediatrics, Medical University of Innsbruck, Anichstraße 35; 6020 Innsbruck, Austria

\begin{abstract}
Introduction: Mitochondrial disorders have a clinical and genetic heterogeneity which often complicates the diagnostic process especially in critically ill patients. Exome sequencing can help to generate a definite diagnosis in a rather short time.

Case Presentation: A boy presented with inspiratory stridor, respiratory insufficiency, progressive muscular hypotonia, poor head control and sucking, and hypersomnia at three months of age. He got ventilator dependent and developed cardiomyopathy within weeks. The progressive multisystem disease leads to the suspicion of a mitochondrial disorder. As neither the clinical nor the metabolic changes or the diagnostic procedures performed seemed indicative of a specific mitochondrial disorder, we performed exome sequencing. This revealed a known homozygous mutation (c.418G>A, p.Glu140Lys) in SCO2.
\end{abstract}

Discussion: Retrospectively, the combination of progressive encephalomyopathy and inspiratory stridor could have been the diagnostic clue for SCO2 deficiency. However, given the enormous clinical and genetic heterogeneity it is nearly impossible to diagnose a mitochondrial disorder with single gene analysis in the restricted time frame clinicians' face when caring for life- threatening ill infants. This case illustrates both the importance of concise clinical characterization and the power of exome sequencing as first line diagnostic procedure.

\section{Introduction}

Mitochondrial disorders are a growing group of hereditary disorders, mutations in more than 280 genes have been related to human disease $[1,2]$. The clinical and genetic heterogeneity complicates the diagnostic process and is especially problematic when facing critically ill patients with progressive disease. Implementation of next generation sequencing techniques, like exome sequencing, into daily clinical routine has revolutionized this process $[1,3]$. Human mitochondrial protein encoded by $\mathrm{SCO} 2$ gene is essential for the assembly process of the respiratory chain complex IV (cytochrome C oxidase, COX), and SCO2 deficiency has been reported to lead to deficiency of COX [4]. SCO2 is needed for copper delivery into the mitochondria [4]. Patients with SCO2 gene mutation have been reported to develop first symptoms at or soon after birth. Individuals often present with severe progressive encephalopathy, muscle hypotonia, lactic acidosis, non-specific findings as seen in many mitochondrial disorders, but often show inspiratory stridor, as a distinctive feature [4-8].

\section{Case Presentation}

After an uneventful pregnancy, a $4 \mathrm{~kg}$ boy of healthy nonconsanguineous caucasian parents was delivered at 40+2 weeks of gestation. The Apgar-scores were 9/10/10. Postnatal adaption was uneventful. At age of 3 months the patient was admitted with an inspiratory stridor without signs of infection. The parents reported prolonged sleeping episodes. The medical examination revealed muscular hypotonia, poor head control and poor sucking. The results of routine blood tests were normal except for an elevated lactate concentration (37.2 mg/dl; ref. range 5-22). Routine testing for inherited metabolic disorders (serum creatinine, ammonia, amino acids in blood and urine and urinary organic acids levels) was unremarkable. Only the alanine concentration in blood was elevated, reflecting the elevated lactate concentration - and possibly indicative for mitochondrial dysfunction. After two weeks, the general condition worsened and the patient developed a 
respiratory insufficiency. Laryngoscopy revealed a partial paralysis of the vocal cords.

The liver function parameters were elevated at ASAT 1,047 U/l (normal range 14-77 U/l), ALAT $774 \mathrm{U} / \mathrm{l}$ (normal range 4-49 $\mathrm{U} / \mathrm{l}$ ). The plasma lactate concentration rose to $101 \mathrm{mg} / \mathrm{dl}$. During the following days the patient developed convulsions requiring the admission to intensive care unit. A tracheostomy was performed. Subsequently, the patient needed mechanical ventilation and anticonvulsive drugs (Figure 1). At age 3 months, the chest X-ray revealed an enlarged heart, the cardiac markers were elevated (creatine kinase $417 \mathrm{U} / \mathrm{l}$ (normal range 41-330), NT-pro BNP
16,163 ng/l (normal range 0-1,000) and troponin-T $66.3 \mathrm{ng} / \mathrm{l}$ (normal range 0-14 ng/l)) and the echocardiography showed a hypertrophic cardiomyopathy with an abnormally structured and thickened myocardium without obstruction (Figure 1). Cerebral magnetic resonance imaging revealed symmetric bilateral changes in the periventricular white matter, the globus pallidus and the capsula interna. Nerve conduction velocity (N. medianus) and electromyography (M. bizeps and M. tibialis anterior) revealed an axonal, sensory and motoric neuropathy. The electroencephalogram showed a slow background activity, diffuse delta-activity and intermittent seizure-patterns (Figure 2).

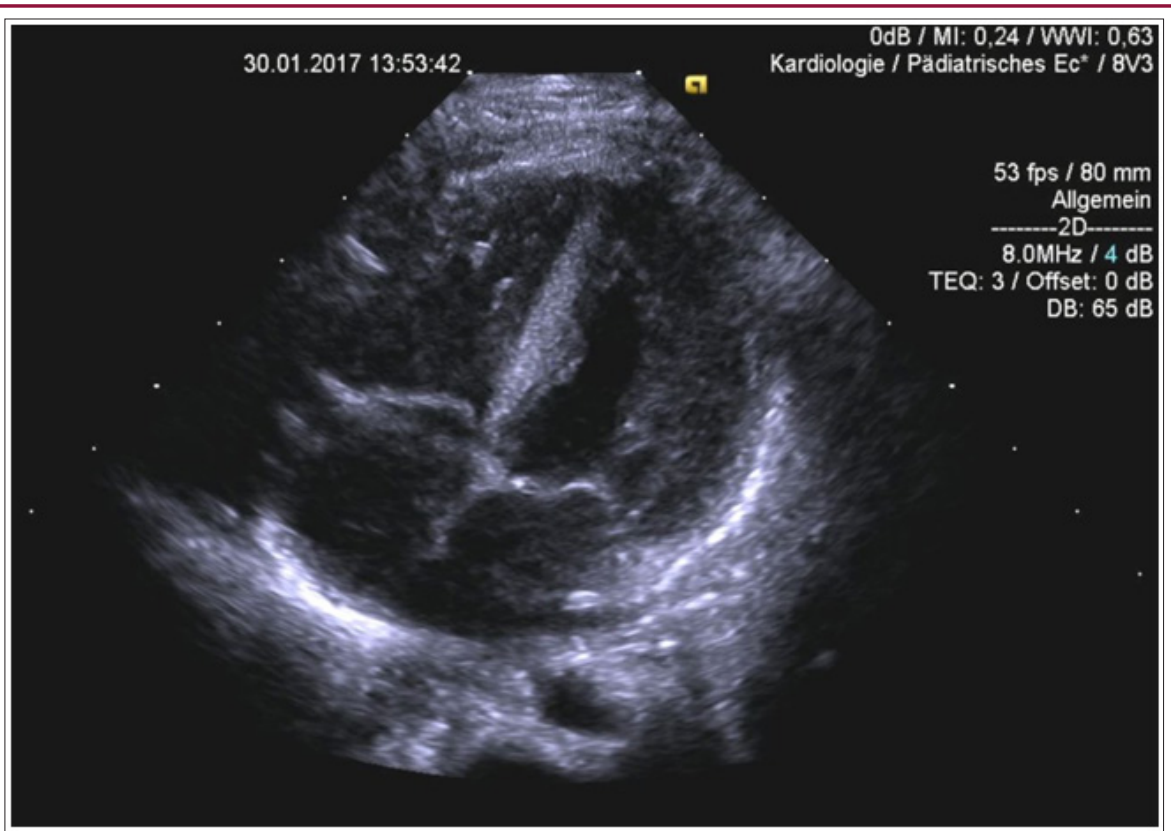

Figure 1: Echocardiography at the age of 3 months showing an abnormal structured and thickened myocardium without any sings of obstruction

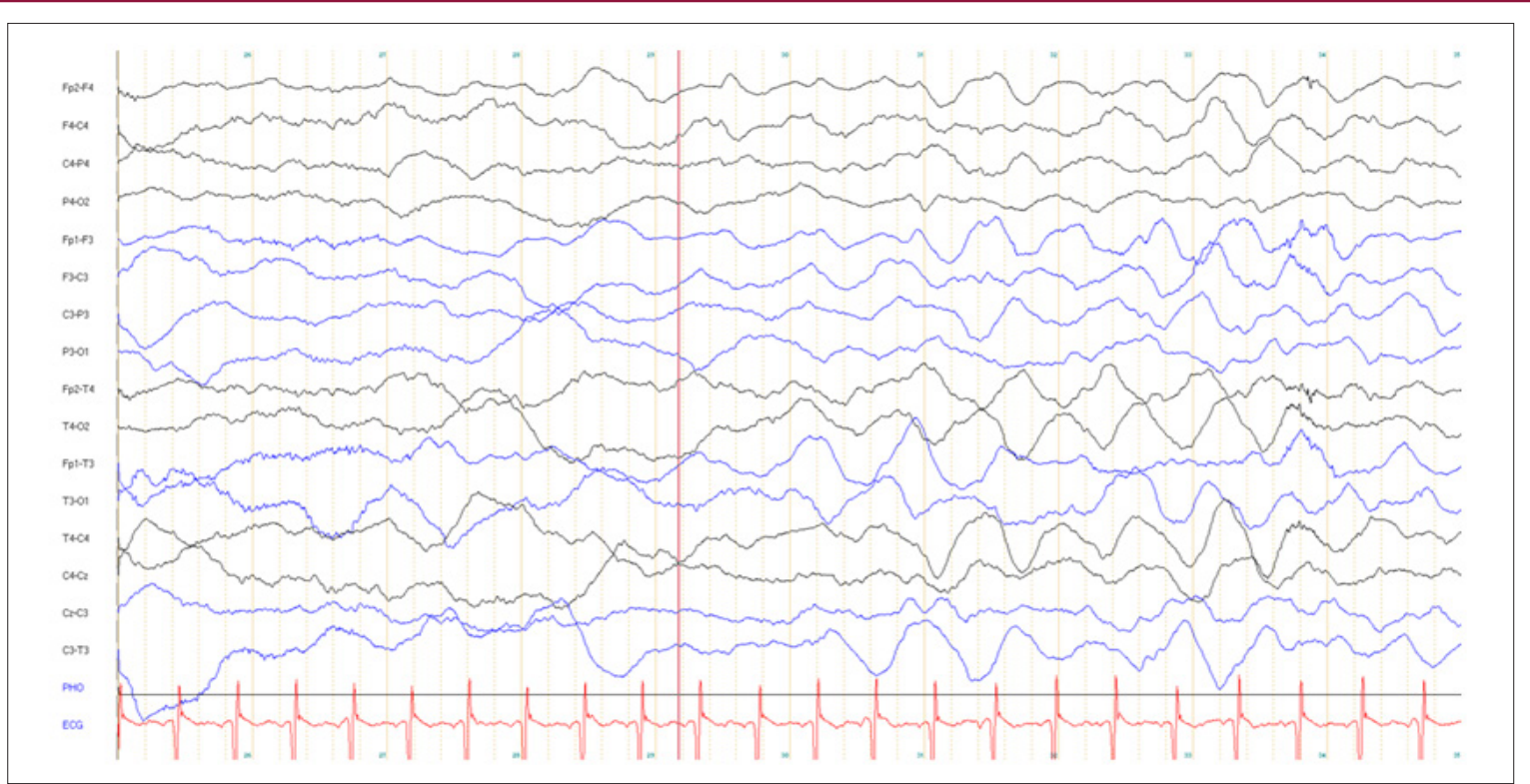

Figure 2: EEG at the age of 4 months showed a slow background activity, diffuse delta-activity and intermittent seizurepatterns. 
The patient needed several anticonvulsive drugs (Pregabalin, Lorazepam, Levetiracetam, Topiramat) to control the daily seizureactivity. Further testing for inherited metabolic disorders (biogene amines, folate and pteridines in csf; isoelectric focussion of transferrin subfractions in plasma; urinay oligosaccharides, mucopolysaccharides, etc.) revealed normal results. However, whole exome sequencing revealed a homozygous mutation of the SCO2 gene (NM_005138.2; c.418G>A, p.Glu140Lys). Both parents are carriers of the same heterozygous mutation on the SCO2 gene. The non-consanguineous parents come from places near to each other in Ukraine. Analysis of enzyme activity of mitochondrial enzymes of the energy homeostasis in fibroblasts revealed a decrease of COX (94 mUnits/mg protein and 138 mUnits/mg protein (normal range: 270-659 mUnits/mg protein). Up to date our patient is on palliative domestic care. He is permanently mechanically ventilated, is fed via PEG tube and receives anticonvulsive drugs. Muscular function has further deteriorated, he cannot move, and does not suck or swallow. On stimulation, he attempts to open his eyes.

\section{Discussion}

We describe a SCO2 mutation in a boy with non-consanguineous parents and an older healthy brother, with first symptoms at the age of 3 months. SCO2 mutations leading to a COX deficiency in central nervous system, heart and skeletal muscle and causing a fatal infantile cardioencephalomyopathy were first described by [7].The SCO2 gene has different functions [7-9]. SCO2 protein is a metallochaperone which is essential for the assembly of the catalytic core of the COX. It firstly acts as an assembly gene required for copper delivery to COX subunit II and is needed for the maintenance of intracellular copper concentrations via COX subunit IV [9]. SCO2 mutations result in very low amounts of COX in skeletal muscle and heart as well as nearly normal concentrations of COX in liver and kidney [4,9]. Up to date, about 50 patients with SCO2 mutations have been described in literature [4,7,9-11]. The characteristic phenotype depends on the mutation detected [9]. Since the first publication of a SCO2 mutation, patients' clinical picture is characterized by cardioencophalomyopathy [7].

During the last years, new SCO2 mutations have been detected mainly causing symptoms of Leigh syndrome, hypertrophic cardiomyopathy, lactic acidosis, inspiratory stridor and respiratory insufficiency and a phenotype similar to patients with a spinal muscular atrophy $[4,7,9-12]$. Our patient showed an early onset respiratory stridor with respiratory insufficiency, an early onset cardiomyeloencephalopathy, peripheral neuropathy and lactic acidosis, thus compiling all the clinical symptoms of SCO2 deficiency. Unfortunately, there is no causative therapy for SCO2 deficiency. Attempts with copper- histidine have been undertaken. Copper-histidine seems to have some beneficial effect. Early supplementation of copper-histidine is assumed to prevent the development of neurological, cardiac and skeletal muscle symptoms [13]. However, the substance does not seem to sufficiently cross the blood brain barrier, and thus has no substantial effect on the CNS symptoms. SCO2 mutation as revealed by several studies is probably not as rare as initially assumed. A retrospective multicenter study on 180 children with a known COX deficiency detected a causative SCO2 mutation in 9 patients [14].

Additionally, another study in 26 children from the Czech and Slovak Republics with a known COX deficiency detected a pathogenic SCO2 mutation in 6 out of 26 patients, confirming that mutations in the SCO2 gene are not as rare as expected [11]. Considering these findings all neonates and infants presenting with muscular hypotonia, cardioencephalomyopathy and inspiratory stridor require an evaluation for multiple congenital syndromes (e.g. inborn disorders of metabolism, mitochondrial disorders, complex genetic disorders, etc.). During the last years clinicians have become more and more aware of genetic determinated diseases like inborn errors of metabolism/mitochondrial diseases. According to the growing awareness mitochondrial diseases have nowadays an estimated incidence of at least 1:5,000 [1]. When suspecting an inborn error of metabolism/mitochondrial disease the clinical and genetic heterogeneity complicates the diagnostic work up. As our case enlightens a precise diagnostic work-up can be unremarkable even when a concise clinical history, the tested laboratory values (lactate, etc.) and the performed medical examination let you first think of a mitochondrial disease or inherited metabolic disorder. In addition to a thorough clinical characterization, further diagnostic steps like whole exome sequencing can be helpful as a step in routine diagnostic work up. In the last decade next-generation sequencing techniques (e.g. exome sequencing) have become widely available $[1,15]$.

Neurological symptoms like progressive muscular weakness and encephalomyopathy can at a first glance mimic the disease. Therefore and as our case highlights, SCO2 gene sequencing may be required to secure the final diagnosis. A recent study focusing on the diagnostic approach of mitochondrial disorders suggested checking for SCO1 and SCO2 mutations in all newborns and infants with cardiomyopathy during diagnostic work up [15]. Summarizing, our case report illustrates that in addition to a thorough clinical characterization, further diagnostic steps like whole exome sequencing can be helpful as a next step in routine diagnostic work up. In patients with neonatal cardiomyopathy and inspiratory stridor, direct testing for $\mathrm{SCO} 2$ mutation can be a diagnostic consideration early in the course of the disease and offers a basic armamentarium when heading for a diagnosis.

\section{References}

1. Wortmann SB, Mayr JA, Nuoffer JM, Prokisch H, Sperl W (2017) A Guideline for the Diagnosis of Pediatric Mitochondrial Disease: The Value of Muscle and Skin Biopsies in the Genetics Era. Neuropediatrics 48(4): 309-314.

2. Skladal D, Sudmeier C, Konstantopoulou V, Stöckler-Ipsiroglu S, PleckoStartinig B, et al. (2003) The clinical spectrum of mitochondrial disease in 75 pediatric patients. Clin Pediatr. (Phila) 42(8): 703-710.

3. Kremer LS, Bader DM, Mertes C, Kopajtich R, Pichler G, et al. (2017) Genetic diagnosis of Mendelian disorders via RNA sequencing. Nat Commun 12: 15824.

4. Verdijk RM, de Krijger R, Schoonderwoerd K, Tiranti V, Smeets H, et al. (2008) Phenotypic consequences of a novel SCO2 gene mutation. Am J Med Genet A 146A(21): 2822-2827. 
5. Pronicki M, Kowalski P, Piekutowska-Abramczuk D, Taybert J, Karkucinska- Wieckowska A, et al. (2010) A homozygous mutation in the SCO2 gene causes a spinal muscular atrophy like presentation with stridor and respiratory insufficiency. Eur J Paediatr Neurol 14(3): 253260.

6. Dickinson EK, Adams DL, Schon EA, Glerum DM (2000) A human SCO2 mutation helps define the role of Sco1p in the cytochrome oxidase assembly pathway. J Biol Chem 275(35): 26780-26785.

7. Papadopoulou LC, Sue CM, Davidson MM, Tanji K, Nishino I, et al. (1999) Fatal infantile cardioencephalomyopathy with COX deficiency and mutations in SCO2, a COX assembly gene. Nat Genet 23(3): 333-337.

8. Gurgel-Giannetti J, Oliveira G, Brasileiro Filho G, Martins P, Vainzof M, et al. (2013) Mitochondrial cardioencephalomyopathy due to a novel SCO2 mutation in a Brazilian patient: case report and literature review. JAMA Neurol 70(2): 258-261

9. Leary SC, Cobine PA, Kaufman BA, Guercin GH, Mattman A, et al. (2007) The human cytochrome c oxidase assembly factors SCO 1 and SCO 2 have regulatory roles in the maintenance of cellular copper homeostasis. Cell Metab 5(1): 9-20.

10. Jaksch M, Ogilvie I, Yao J, Kortenhaus G, Bresser HG, et al. (2000) Mutations in SCO2 are associated with a distinct form of hypertrophic cardiomyopathy and cytochrome c oxidase deficiency. Hum Mol Genet. 9(5):795-801.

11. Vesela K, Hansikova H, Tesarova M, Martasek P, Elleder M, et al. (2004) Clinical, biochemical and molecular analyses of six patients with isolated cytochrome c oxidase deficiency due to mutations in the SCO2 gene. Acta Paediatr 93(10): 1312-1317.

12. Salviati L, Sacconi S, Rasalan MM, Kronn DF, Braun A, et al. (2002) Cytochrome c oxidase deficiency due to a novel $\mathrm{SCO}_{2}$ mutation mimics Werdnig-Hoffmann disease. Arch Neurol 59(5): 862-865.

13. Freisinger P, Horvath R, Macmillan C, Peters J, Jaksch M (2004) Reversion of hypertrophic cardiomyopathy in a patient with deficiency of the mitochondrial copper binding protein Sco2: is there a potential effect of copper? J Inherit Metab Dis 27(1): 67- 79.

14. Böhm M, Pronicka E, Karczmarewicz E, Pronicki M, PiekutowskaAbramczuk D, et al. (2006) Retrospective, multicentric study of 180 children with cytochrome C oxidase deficiency. Pediatr Res 59(1): 21-26.

15. Honzik T, Tesarova M, Magner M, Mayr J, Jesina P, et al. (2012) Neonatal onset of mitochondrial disorders in 129 patients: clinical and laboratory characteristics and a new approach to diagnosis. J Inherit Metab Dis 35(5): 749-759.
(C) Commons Attribution 4.0 License

Submission Link: https://biomedres.us/submit-manuscript.php

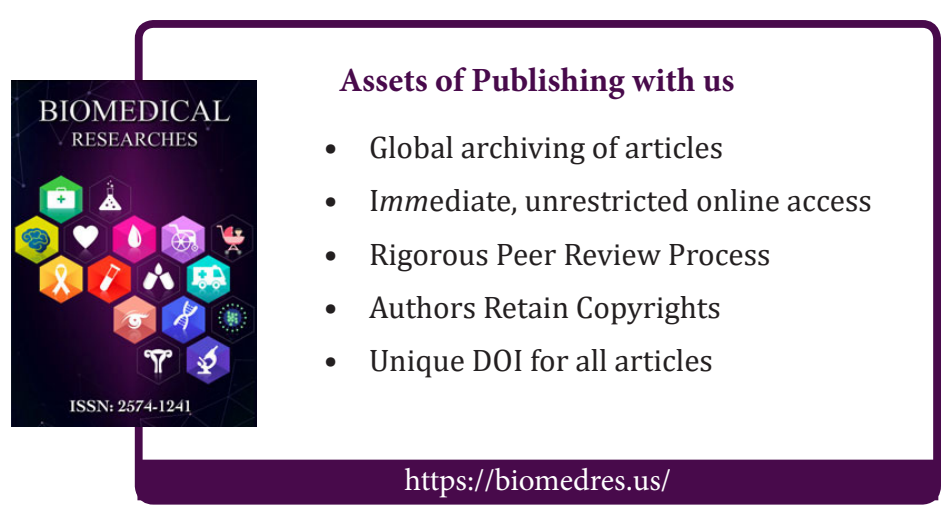

